\title{
THE IMPACT OF PRE-EXISTING AND POST-TRANSPLANT DIABETES MELLITUS ON OUTCOMES FOLLOWING LIVER TRANSPLANTATION
}

\author{
Aloysious D Aravinthan 1,2,3, Waleed Fateen 2,3, Adam C Doyle ${ }^{1,4}$, Suresh V Venkatachalapathy ${ }^{3}$, \\ Assaf Issachar ${ }^{1,5}$, Zita Galvin ${ }^{1}$, Gonzalo Sapisochin ${ }^{1}$, Mark S Cattral ${ }^{1}$, Anand Ghanekar ${ }^{1}$, Ian D \\ McGilvray ${ }^{1}$, Markus Selzner ${ }^{1}$, David R Grant ${ }^{1}$, Paul D Greig ${ }^{1}$, Nazia Selzner ${ }^{1}$, Leslie B Lilly ${ }^{1}$, Eberhard \\ L Renner $^{1,6}$, Mamatha Bhat ${ }^{1}$ \\ ${ }^{1}$ Multiorgan Transplant Program, Toronto General Hospital, University of Toronto, Toronto, Canada \\ ${ }^{2}$ Nottingham Digestive Diseases Centre, School of Medicine, University of Nottingham, Nottingham, \\ ${ }^{3}$ National Institute for Health Research (NIHR) Nottingham Biomedical Research Centre, Nottingham \\ University Hospitals NHS Trust and University of Nottingham, Nottingham, UK \\ 4Department of Gastroenterology and Hepatology, Royal Perth Hospital, Australia \\ ${ }^{5}$ Liver Institute, Rabin Medical Center, Beilinson Hospital, Petah Tikva, Israel \\ ${ }^{6}$ Department of Medicine, Max Rady College of Medicine/Rady Faculty of Health Sciences, University \\ of Manitoba, Winnipeg, MB, Canada
} UK 


\section{ABSTRACT}

Background: Diabetes mellitus (DM) is said to adversely affect transplant outcomes. The aim of this study was to investigate the impact of pre-existing and post-transplant DM on liver transplant (LT) recipients.

Method: A single centre retrospective analysis of prospectively collected data of LT recipients (19902015) was undertaken.

Results: Of the 2,209 patients, $13 \%(n=298)$ had Pre-DM, $16 \%(n=362)$ developed PTDM, $5 \%(n=118)$ developed transient hyperglycemia ( $t-H G)$ post-LT, and $65 \%(n=1,431)$ never developed DM (no DM). Baseline clinical characteristics of patients with PTDM was similar to that of patients with pre-DM. Incidence of PTDM peaked during first-year (87\%) and plateaued thereafter. On multivariate analysis (Bonferroni-corrected), non-alcoholic fatty liver disease and the use of Tacrolimus and Sirolimus use were independently associated with PTDM development. Both Pre-DM and PTDM patients had satisfactory and comparable glycaemic control throughout the follow-up period. Those who developed t-HG seems to have a unique characteristic compared to others. Overall, $9 \%, 5 \%$, and $8 \%$ developed end-stage renal disease (ESRD), major cardiovascular event (mCVE), and de novo cancer, respectively. Both Pre-DM and PTDM did not adversely affect patient survival, re-LT, or de novo cancer. The risks of ESRD and mCVE were significantly higher in patients with Pre-DM followed by PTDM and no DM.

Conclusions: In this largest non-registry study, patients with pre-DM and PTDM share similar baseline clinical characteristics. Pre-DM increases the risk of ESRD and $\mathrm{mCVE}_{\text {; }}$ however, patient survival was comparable to those with PTDM and without diabetes. Understanding the impact of PTDM would need prolonged follow-up. 


\section{INTRODUCTION}

Diabetes Mellitus (DM) poses an increasing challenge for patients and health care systems worldwide [1]. It leads to significant morbidity and mortality through several macrovascular and microvascular complications [2, 3]. Further, DM is also associated with an increased risk of several cancers [4]. DM is an important risk factor for the development of non-alcoholic fatty liver disease (NAFLD), which is a leading cause of cirrhosis, liver cancer, and liver transplantation (LT). Further, the presence of DM increases the risk of death in patients with liver disease [5].

LT remains the only treatment option that improves the dismal prognosis of end-stage liver disease. Advances in perioperative management and immunosuppressive treatment has led to excellent longterm outcomes with $1-, 5-$, and 10 -year post-LT survival rates of $>90 \%, 85 \%$, and $70 \%$, respectively [6-8]. However, post-transplant immunosuppression remains associated with a number of side effects including new-onset diabetes mellitus known as post-transplant diabetes mellitus (PTDM). PTDM was first described over 50 years ago [9], but has only more recently gained recognition as a major complication with the potential for serious consequences [10]. The reported incidence of PTDM after all solid organ transplantation ranges from $2 \%$ to $53 \%[10,11]$. PTDM has been reported in $7-28 \%$ of LT recipients LT [12]. While the pathophysiology of PTDM is not fully understood, pancreatic $\beta$-cell dysfunction, impaired insulin secretion (associated with the use calcineurin inhibitors and mammalian target of rapamycin complex 1 inhibitors), postoperative weight gain and hepatitis C virus infection (associated with insulin resistance) are all thought to play a role $[13,14]$.

The impact of PTDM and pre-existing DM on outcomes following LT is limited and equivocal. While some studies demonstrate a negative impact of PTDM on patient and graft survival [15-20], others were not able to confirm this [21-23]. Further, these studies have usually not compared the impact of PTDM to that of pre-existing DM $[15,17,18,22]$, and many had limited follow-up duration potentially leading to underestimating of a potential impact $[16,18,21,22]$. In addition, most of these studies have been reported from East Asia $[15,17,18,21,23]$, and therefore may not represent the experience in the West. The aim of the present study is to assess the impact of pre-existing DM and PTDM on long-term outcomes of LT recipients in the Toronto transplant program, one of the largest in North America. 


\section{MATERIALS \& METHODS}

\section{Patient selection and data collection}

This was a retrospective analysis of prospectively collected data from a single, high-volume liver transplant center. All adult patients who received a liver transplant in the Toronto liver transplant program for any indication between January 1990 and December 2015 were eligible for inclusion. The following patients were excluded from the study: (a) patients who underwent combined solid organ transplantation, and (b) patients who were transplanted in other centers and subsequently followed up in the Toronto program.

Demographic and clinical data were extracted from electronic transplant database (OTTR: Transplant Care Platform 6, OTTR Chronic Care Solutions, Omaha, NE), and supplemented with manual chart review.

For the purposes of this study, patients were categorised into four groups based on diabetic status: those without DM before and after transplantation (no DM); those with pre-transplant DM (Pre-DM); those who developed PTDM; and those who developed transient hyperglycemia (t-HG). Main analysis included no DM, Pre-DM, and PTDM groups. t-HG group was compared to the other three groups separately but was not included in the main analysis.

The study was approved by the Toronto University Health Network Research Ethics Board (REB 165023).

\section{Diagnosis of PTDM}

PTDM was diagnosed, based on the 2003 International Consensus Guidelines [24]. LT recipients who were not diabetic prior to transplantation but developed symptoms of DM with an elevated random plasma glucose $(\geq 11.1 \mathrm{mmol} / \mathrm{l})$ or an elevated fasting plasma glucose $(\geq 7.0 \mathrm{mmol} / \mathrm{l})$ or an elevated 2hour plasma glucose ( $\geq 11.1 \mathrm{mmol} / \mathrm{l})$ during an oral glucose tolerance test were diagnosed to have PTDM. In addition, those who were initiated on hypoglycemic agents for more than 14 continuous days post-transplant were also considered to have PTDM. Glucose levels are monitored daily (preand post-meal) during the immediate post-transplant period. Following discharge, random glucose level is regularly checked in LT recipients at their out-patient clinic appointments and those with elevated glucose levels are referred to their family physicians for further investigations.

Those who diagnosed with DM range hyperglycaemia based on the above criteria, but became euglycemic without the need for hypoglycemic agents within 6 months of LT were categorised as tHG.

\section{Immunosuppression regimen}

All patients received intravenous Methylprednisolone followed by an oral course of Prednisone and Tacrolimus immediately following LT; Basiliximab (before that thymoglobulin) induction was used to delay introduction of calcineurin inhibitors in some cases (e.g. patients with renal failure). Oral Prednisone dose followed a standard tapering regimen and was stopped at 3 months post-LT. Tacrolimus was substituted with Cyclosporine in case of Tacrolimus intolerance or neurotoxicity. Mycophenolate mofetil was added if calcineurin inhibitor (Tacrolimus or Cyclosporine) dose reduction was required. Long-term dual immunosuppressant regimen (calcineurin inhibitor or Sirolimus, and Mycophenolate mofetil) was the standard of care for patients transplanted for 
autoimmune liver diseases, acute liver failure, and inborn metabolic disorders. Long-term low-dose Prednisone was part of the immunosuppressant regimen for patient with a history of recurrent acute cellular rejection or chronic rejection. Further, the target trough levels of Cyclosporine, Tacrolimus, and Sirolimus remained unchanged over the study period. However, the use of immunosuppressants evolved during the 25 -year study period. In brief, these include introduction of Tacrolimus as the primary immunosuppressant in 2001/2002, prior to which Cyclosporine remained the primary immunosuppressant and the use of Sirolimus since 2005 as the primary immunosuppressant in those who were intolerant to calcineurin inhibitors.

For the purposes of this study, only those patients who were taking an immunosuppressant continuously for a minimum of 6 months were considered to have a positive exposure to that immunosuppressant.

\section{Endpoints}

Patient survival was the primary endpoint. Patient survival was defined as the time from LT to death from any cause. Patients who were lost to follow-up, or moved to another center for ongoing care, were censored at the time of their last clinic visit. In the event of more than one $L T$, cases were censored at the time of the second LT.

Re-LT and development of end-stage renal disease (ESRD), major cardiovascular events (mCVE), and de novo cancer were secondary endpoints. ESRD was defined as GFR $<15 \mathrm{ml} / \mathrm{min} / 1.73 \mathrm{~m}^{2}$ for $6 \mathrm{months}$ or more, requirement of renal replacement therapy, or renal transplantation. Myocardial infarction and stroke were considered as mCVE.

\section{Data analysis}

Continuous variables were presented as median and interquartile range (IOR). Categorical variables were presented as number and percentage. Normality of distributions was assessed using histograms and confirmed using Shapiro-Wilk test. Kruskal-Wallis, Mann-Whitney and Chi-square test were used as appropriate to compare basic demographics of the four study groups. A p-value of $<0.05$ was considered significant.

Predictors of PTDM was investigated using univariate and multivariate analysis. Univariate analysis was performed using Mann-Whitney test and Chi-square for continuous and categorical variables, respectively. Variables with a $p$-value $<0.10$ were included in the multivariate logistic regression model. Variables were considered to have independent association only if the $p$-value reached Bonferroni-corrected level of significance.

Patient survival analysis for all-cause mortality (primary outcome) was performed using the KaplanMeier method. The probability of re-LT and developing ESRD, $\mathrm{MCVE}$, and de novo cancer were investigated using a competing risk model. Death was considered as a competing event and the cumulative incidence of re-LT, ESRD, mCVE, and de novo cancer were calculated using Gray's test.

Statistical analyses were performed using IBM SPSS Statistics for Windows, Version 20.0 (Armonk, NY) for univariate and multivariate prediction of PTDM, GraphPad prism 5 (San Diego, CA) for Kaplan-Mayer analysis, and visualisation and $\mathrm{R}$ statistical software, version 3.4.1 (http://cran.r- 
project.org/) for competing risk analysis. The R-package "cmprsk" version 2.2-7 was used to compare the cumulative incidence and conduct a regression analysis of competing risks. 


\section{RESULTS}

\section{Demographics and clinical characteristics}

The study included 2,209 patients, who underwent LT between January 1990 and December 2015 and fulfilled the eligibility criteria. The median age at LT was 54 years (IOR $47-60)$ and $67 \%(n=1,480)$ were males (table 1). The median duration of post-LT follow-up was 6.7 years (IOR 2.9-11.3).

Of the 2,209 patients, $13 \%$ ( $n=298$; median follow-up 6.3 years [IOR $2.7-10.4$ ]) had Pre-DM, $16 \%$ $(n=362$; median follow-up 8.9 years [IOR 4.9-12.7]) developed PTDM, 5\% ( $n=118$; median follow-up 6.4 years [IOR $1.6-10.8$ ]) developed $t-H G$, and $65 \%(n=1,431$; median follow-up 6.1 years [IOR $2.8-$ 10.9]) remained free of DM. The demographic characteristics at LT of all four groups are summarised in table 1.

\section{Incidence, risk factors, and characteristics of PTDM}

Of the 1,911 patients who were not diabetic at LT, 362 (19\%) developed PTDM following LT. The vast majority were diagnosed to have PTDM during the first year after $L T(87 \%, n=314)$; the occurrence of PTDM plateaued thereafter -8 per year at 2 years, 3 per year at 5 years, and 2 per year at 10 years post-LT. Further, the era of transplantation did not impact the development of PTDM $-21 \%(n=77)$ of 374 patients who were transplanted in $1990-1999,19 \%(n=171)$ of 916 patients who were transplanted in 2000-2009, and 18\% ( $n=114)$ of 621 patients who were transplanted in 2010-2015 developed PTDM $(p=0.66)$.

In comparison to no DM group, the following parameters were associated with the development of PTDM (table 2). On univariate analysis, age, male sex, BMI, non-alcoholic fatty liver disease as the etiology of liver disease, pre-LT dyslipidemia, cold ischemia time, RouX-en-Y biliary anastomosis, and the use of Cyclosporin, Sirolimus, and Prednisone for immunosuppression. In addition to the above parameters, pre-LT hypertension $(p=0.07)$ and the use of Tacrolimus $(p=0.06)$ and Mycophenolate $(p=0.07)$ were included in the multivariate analysis (total of 13 parameters); the Bonferroni-corrected level of significance in the multivariate analysis was $p<0.0038$. On multivariate (Bonferronicorrected) analysis, non-alcoholic fatty liver disease as the etiology of liver disease (OR $2.03,95 \% \mathrm{Cl}$ 1.31-3.16; $\mathrm{p}=0.002$ ) and the use of Tacrolimus (OR 2.76, 95\% $\mathrm{Cl} 1.78-4.30 ; \mathrm{p}<0.001$ ) and Sirolimus (OR $2.72,95 \% \mathrm{Cl} 1.67-4.42, \mathrm{p}<0.001$ ) as immunosuppression were independently associated with the development of PTDM.

The clinical characteristics of PTDM patients at LT were similar to Pre-DM patients but were different to that of no DM patients. Compared to no DM group, PTDM patients were older $(p<0.0001)$, predominantly male $(p=0.001)$, with higher BMI $(p<0.0001)$, and were likely to have non-alcoholic fatty liver disease as the etiology of liver disease ( $p<0.0001)$. In contrast, PTDM patients had marked similarities with Pre-DM patients at LT - age $(p=0.40)$, sex $(p=0.09)$, BMI $(p=0.99)$, and non-alcoholic fatty liver disease etiology $(p=0.54)$. Interestingly, hepatitis $C$ etiology did not differ between PTDM and no DM $(p=0.21)$ or Pre-DM $(p=0.23)$ groups.

Both PTDM and Pre-DM patients had optimal and comparable glycemic control during the post-LT period. The overall median glycosylated hemoglobin (HbA1c) of PTDM and Pre-DM were 6.7\% (IQR $6.4-7.0$ ) and $6.9 \%$ (IOR $6.7-7.5)$, respectively. Similarly, the yearly median HbA1c ranged between $6.0 \%-7.1 \%$ and $6.3 \%-8.1 \%$, respectively (figure 1 ). 


\section{Primary outcome (patient survival)}

Over a third ( $\mathrm{n}=782,35 \%)$ of the total study population died following LT. The 1-, 5-, 10-, and 20-year patient survival rates were $90 \%, 79 \%, 64 \%$, and $23 \%$ in the Pre-DM group, $97 \%, 88 \%, 74 \%$, and $22 \%$ in the PTDM group, and $90 \%, 79 \%, 68 \%$, and $34 \%$ in the no-DM group, respectively. Overall, there was no difference in patient survival between the three study groups ( $p=0.30$, figure 2 ). A summary of the major causes of death are shown in Supplementary Table 1.

\section{Secondary outcomes}

\section{$\operatorname{Re}-L T$}

Overall, 6\% $(n=126)$ underwent re-LT. Of the 298 patients with Pre-DM, 4\% $(n=12)$ underwent re-LT, $32 \%(n=94)$ died without undergoing re-LT, and $64 \%(n=192)$ were alive without requiring re-LT. The $1-, 5-, 10-$, and 20 -year cumulative incidence of re-LT were $3 \%, 4 \%, 5 \%$, and $5 \%$, respectively. Of the 362 patients with PTDM, 6\% $(n=23)$ underwent re-LT, 35\% $(n=127)$ died without undergoing re-LT, and $59 \%(\mathrm{n}=212)$ were alive without requiring re-LT. The 1-, 5-, 10-, and 20-year cumulative incidence of re-LT were $2 \%, 3 \%, 4 \%$, and $10 \%$, respectively. Similarly, of the 1,431 patients with no DM, $6 \%(n=91)$ underwent re-LT, 33\% ( $n=467)$ died without undergoing re-LT, and $61 \%(n=873)$ were alive without requiring re-LT. The 1-, 5-, 10-, and 20-year cumulative incidence of re-LT were $3.1 \%, 4.6 \%, 6.6 \%$, and $9.3 \%$, respectively. The cumulative incidence of re-LT was similar between the three study groups $(p=0.50)$.

\section{$\underline{E S R D}$}

Overall, $9 \%(n=194)$ developed ESRD following LT. Of the 298 patients with Pre-DM, $12 \%(n=35)$ developed ESRD, $28 \%(n=82)$ died without developing ESRD, and $61 \%(n=181)$ were alive and free of ESRD. The 1-, 5-, 10-, and 20-year cumulative incidence of ESRD were $3 \%, 7 \%, 13 \%$, and $39 \%$, respectively. Of the 362 patients with PTDM, 10\% $(n=38)$ developed ESRD, 32\% $(n=117)$ died without developing ESRD, and $57 \%(n=207)$ were alive and free of ESRD. The 1-, 5-, 10-, and 20-year cumulative incidence of ESRD were $1 \%, 4 \%, 8 \%$, and $22 \%$, respectively. Similarly, of the 1,431 patients with no DM, 7.6\% ( $n=109)$ developed ESRD, 31.2\% $(n=447)$ died without developing ESRD, and $61.1 \%$ ( $n=875$ ) were alive and free of ESRD (median follow up 8.0 years). The 1-, 5-, 10-, and 20year cumulative incidence of ESRD were $2 \%, 4 \%, 7 \%$, and $15 \%$, respectively. The cumulative incidence of developing ESRD was significantly higher in Pre-DM patients compared to no DM patients $(p=0.003)$ but was similar to that of PTDM patients $(p=0.08)$. However, the cumulative incidence of developing ESRD was similar in patients with PTDM and no DM $(p=0.27)$.

\section{$\underline{m C V E}$}

Overall, 5\% ( $n=103)$ developed mCVE following LT. Of the 298 patients with Pre-DM, 7\% $(n=21)$ experienced a $m C V E, 32 \%(n=94)$ died without ever experiencing a $m C V E$, and $61 \%(n=183)$ were alive and free of mCVE. The 1-, 5-, 10-, and 20-year cumulative incidence of mCVE were $2 \%, 3 \%, 8 \%$, and $15 \%$, respectively. Of the 362 patients with PTDM, 6\% $(n=21)$ experienced a $m C V E, 34 \%(n=123)$ died without ever experiencing a $\mathrm{mCVE}$, and $60 \%(n=218)$ were alive and free of $\mathrm{mCVE}$. The $1-, 5^{-}, 10-$, and 20 -year cumulative incidence of $\mathrm{mCVE}$ were $1 \%, 2 \%, 4 \%$, and $10 \%$, respectively. Similarly, of the 1,431 patients with no DM, 4\% $(n=57)$ experienced a mCVE, 33\% $(n=475)$ died without ever experiencing a $\mathrm{mCVE}$, and $63 \%(\mathrm{n}=899)$ were alive and free of $\mathrm{mCVE}$. The 1-, 5-, 10-, and 20-year 
cumulative incidence of $\mathrm{mCVE}$ were $1 \%, 2 \%, 4 \%$, and $7 \%$, respectively. The cumulative incidence of developing $\mathrm{mCVE}$ was significantly higher in Pre-DM patients compared to no DM patients $(p<0.01)$ but was similar to that of PTDM patients $(p=0.11)$. However, the cumulative incidence of developing $m C V E$ was similar in PTDM and no DM patients $(p=0.95)$.

\section{De novo cancer}

Overall, $8 \%(n=183)$ developed de novo cancer following LT. Of the 298 patients with Pre-DM, $9 \%$ $(n=26)$ developed de novo cancer, $31 \%(n=93)$ died without developing de novo cancer, and $60 \%$ $(n=179)$ were alive and free of de novo cancer. The 1-, 5-, 10-, and 20-year cumulative incidence of de novo cancer were $2 \%, 7 \%, 10 \%$, and $13 \%$, respectively. Of the 362 patients with PTDM, $9 \%(n=33)$ developed de novo cancer, $31 \%(n=112)$ died without developing de novo cancer, and $60 \%(n=217)$ were alive and free of de novo cancer. The 1-, 5-, 10-, and 20-year cumulative incidence of de novo cancer were $2 \%, 5 \%, 8 \%$, and $14 \%$, respectively. Similarly, of the 1,431 patients with no DM, $8 \%$ $(n=116)$ developed de novo cancer, $31 \%(n=450)$ died without developing de novo cancer, and $60 \%$ $(\mathrm{n}=865)$ were alive and free of de novo cancer. The 1-, 5-, 10-, and 20-year cumulative incidence of de novo cancer were $1 \%, 4 \%, 7 \%$, and $15 \%$, respectively. Unlike with ESRD and $\mathrm{mCVE}$, the cumulative incidence of developing de novo cancer in no DM patients was similar to that of Pre-DM $(p=0.43)$ and PTDM ( $p=0.95)$ patients. The distribution of different types of cancers within each group is summarised in Supplementary Table 2.

\section{Transient hyperglycaemia}

One hundred and eighteen patients fulfilled the diagnostic criteria of t-HG. All achieved a state of euglycemia without the need for anti-diabetic medications within 6 months of LT.

$\mathrm{t}-\mathrm{HG}$ patients were unique in their baseline clinical characteristics. The age of $\mathrm{t}-\mathrm{HG}$ patients at LT was no different from that of patients with Pre-DM $(p=0.06)$, PTDM $(p=0.25)$, and no DM $(p=0.11)$. However, BMI of t-HG patients at LT was significantly higher than that of no DM patients $(p=0.03)$ but was similar to that of Pre-DM $(p=0.50)$ and PTDM $(p=0.54)$ patients. Similarly, $t-H G$ patients were predominantly males compared to no DM patients $(p=0.01)$ but were not different from Pre-DM $(p=0.47)$ or PTDM $(p=0.60)$ patients. Yet, the prevalence of NAFLD being the underlying etiology of liver disease was similar between $\mathrm{t}-\mathrm{HG}$ and no $\mathrm{DM}(p=0.36)$ patients but was significantly lower compared to that of Pre-DM $(p=0.0006)$ and PTDM $(p=0.002)$ patients. More interestingly, the prevalence of hepatitis $C$ being the underlying etiology of liver disease was significantly higher in $t-$ HG patients compared to no DM $(p=0.002)$ and PTDM $(p=0.04)$ patients but similar to that of Pre-DM $(p=0.25)$ patients.

Outcomes of patients with t-HG were not analysed due to small number of events. 


\section{DISCUSSION}

This study represents the largest reported non-registry long-term experience on DM (Pre-DM and PTDM) following LT to date. Further, it represents a multi-ethnic Western population, whereas previous literature predominantly involves studies of monoethnic cohorts from East Asia. Additionally, this study explores the risk factors of PTDM and the impact of Pre-DM and PTDM on renal, cardiovascular, and de novo cancer outcomes. Neither the Pre-DM nor the PTDM appear to impact patient survival, re-transplantation, or development of de novo cancer. However, not surprisingly, the risk of developing ESRD and mCVE was significantly higher in patients with Pre-DM. Although not statistically significant, the impact of PTDM on ESRD and mCVE was numerically between that of Pre-DM and no DM.

A quarter of the patients who were not diabetic at the time of LT developed PTDM, which is on par with the previously reported $19-35 \%[15,17,18,25-28]$. A few previous studies however have reported a lower incidence rate $(6-8 \%)[21,29]$ and the reason/s for this substantial difference is not apparent. Further, the occurrence of PTDM was considerably high during the early post-transplant period in this study and previous studies $[18,21,26,27]$. In addition, the demographic and clinical characteristics at the time of LT of patients with Pre-DM and patients who later developed PTDM were similar. This remarkable similarity and the onset of PTDM immediately following LT raises important questions including whether PTDM is merely the clinical manifestation of a previously subclinical prediabetic state and whether those at risk of developing PTDM may potentially be identified pre-transplant with rigorous investigations such as oral glucose tolerance test or glucose clamp studies. The above notions are perhaps supported by the findings of a retrospective study [30], which suggest a twofold increase in the development of PTDM with every 1omg/dl increase in pretransplant fasting plasma glucose level. However, prospective studies are needed for the validation of these hypotheses.

A wide variety of recipient and donor factors, etiology and severity of liver disease, and surgical and post-transplant management factors have been suggested to play a role in the development of PTDM $[15,17,18,21,25-33]$, with limited consistency in common independent risk factors. The possible explanations for such discrepancy between studies include difference in cohort characteristics, cohort size, follow-up duration, and factors included in the analysis. A recent meta-analysis involving over 4,500 patients from 19 retrospective studies identified male sex, body mass index, hepatitis $C$ etiology, pre-LT impaired fasting glucose, and use of tacrolimus as independent risk factors of PTDM [34]. However, this meta-analysis failed to include recipient age, non-alcoholic fatty liver disease etiology, and donor factors in the analysis, which have been shown to increase the risk of PTDM development. Although we included a range of factors that were shown to be associated with the development of PTDM in our analysis, Bonferroni correction was undertaken to mitigate the problem of multiple comparisons. In our study, NAFLD etiology and the use of Sirolimus and Tacrolimus were the only independent factors associated with the development of PTDM. Sirolimus inhibits insulinmediated inactivation of hepatic glycogenolysis [35], causes a reduction in human pancreatic ductal cells, and inhibits glucose-stimulated insulin secretion [36], which potentially explains its role in the development of PTDM. Similarly, reduced insulin secretion and increased insulin resistance have been proposed as potential mechanisms of Tacrolimus-related PTDM [37]. On the other hand, the independent association between NAFLD etiology and PTDM, may be due to the subclinical prediabetic status of these individuals (as discussed above). 
Interestingly, neither the Pre-DM nor the PTDM adversely impacted patient survival and retransplantation. However, despite the optimal HbA1c control in the post-LT period, Pre-DM adversely affected renal and cardiovascular outcomes. The possible reasons for this are the presence of micro and macrovascular end-organ damage even prior to the formal diagnosis of DM [38] and the questionable reliability of $\mathrm{HbA} 1 \mathrm{c}$ as a marker of glycemic control [39]. On the other hand, the impact of PTDM on ESRD and mCVE was in between that of Pre-DM and no DM. Given that the atherosclerosis which leads to both micro and macrovascular complications require prolonged duration of DM, we suspect that PTDM would have an adverse impact on renal and cardiovascular outcomes that is comparable to that of Pre-DM in the long term. These findings enhance our understanding of Pre-DM and PTDM and their effect on the quality and quantity of life after LT, which is helpful especially given the paucity of such data to date. However, given that the use of Tacrolimus was an independent risk factor for PTDM, the development of ESRD especially in patients with PTDM may have be confounded by the use of Tacrolimus. On the other hand, as we enter an era where recurrent hepatitis $\mathrm{C}$ after $\mathrm{LT}$ becomes obsolete, and de novo/recurrent NAFLD increase in incidence, we are likely to see changes in the patterns of incidence and impact of PTDM.

The literature on the impact of both Pre-DM and PTDM, on outcomes in other solid organ transplantation has been limited and contradictory. In a South Korean study of 176 kidney transplant recipients without diabetes at transplantation, PTDM was associated with the increased incidence of cardiovascular disease but did not compromise graft or patient survival [40]. In a single-centre study from Portugal involving 648 kidney transplant recipients, PTDM was shown to have no impact on renal dysfunction, cardiovascular events, graft, and patient survival [41]. Similarly, in a study from South Africa involving 111 kidney transplant recipients, PTDM did not impact graft or patient survival [42]. However, in a multicentre study involving over 2000 kidney transplant recipients from five North American and two European centres, PTDM was independently associated with graft survival; this study failed to investigate the impact of PTDM on patient survival or other outcomes [43]. In a registry study involving more than 9,00o lung transplant recipients, both Pre-DM and PTDM were shown to impact patient survival adversely [44]. Literature on the impact of Pre-DM and PTDM on outcomes heart transplant recipients were not available.

Interestingly, those who developed transient hyperglycaemia (t-HG patients) had unique demographic and clinical characteristics, with hepatitis $C$ being the most common underlying etiology of liver disease in these patients; prevalence of NAFLD was at its lowest despite increased $B M I$. Whether hepatitis $C$ played a crucial role in the development of transient hyperglycemia during the immediate post-LT period and why the hyperglycemic state did not continue beyond the first 3 months remain open to speculation.

Our study has its own strengths and limitations. It is the largest non-registry study to date. Unlike the national registry studies, single-center studies, such as ours, have the advantage of consistency with respect to the use of immunosuppression and the management of DM. Our study also has the advantage of representing the longest duration of post-LT follow-up, with up to 20 years after transplantation; previous studies have been restricted with follow-up and have been limited by relatively small patient numbers. Further, ours is the first non-registry study to investigate the impact on the development ESRD, major cardiovascular events, and de novo cancer in liver transplant 
recipients with Pre-DM, PTDM and no DM, in the long term. On the other hand, the retrospective nature of our study is a natural limitation, due to the risk of bias inherent to study design when compared to a prospective trial. One such limitation is the lack of data on the duration of DM in PreDM patients, which has limited our ability to distinguish those who developed DM secondary to cirrhosis from those with DM that is unrelated to cirrhosis. Interestingly, neither the BMI at listing nor the change in BMI during the first year of transplantation were associated with development of PTDM. This, however, needs to be interpreted with caution because of the limitations in the accuracy of BMI measurement in the pre-transplant population (e.g. due to fluid retention). Further, though our study represents one of the longest experiences, it may still not be adequate to capture the 'true' impact of PTDM on outcomes.

In conclusion, this is the largest non-registry study to investigate the impact of Pre-DM and PTDM on LT outcomes. The baseline (at LT) clinical characteristics of patients who later developed PTDM were similar to that of patients with Pre-DM, suggesting that the genetic susceptibility may be similar between the two clinical entities. Pre-DM seem to pose the highest risk towards the development of ESRD and $m C V E$ following $L T$, despite satisfactory $\mathrm{HbA} 1 \mathrm{c}$ control. This emphasises the urgent need for better investigation/s of glycaemic control to minimize the long-term impact of DM. Although PTDM appears to have a lesser impact than that of Pre-DM, this needs to be interpreted with caution as the pathogenesis of macrovascular and microvascular complications requires prolonged duration of DM. 


\section{REFERENCES}

1. Kanter, J.E. and K.E. Bornfeldt, Impact of Diabetes Mellitus. Arterioscler Thromb Vasc Biol, 2016. 36(6): p. 1049-53.

2. Narres, M., et al., The Incidence of End-Stage Renal Disease in the Diabetic (Compared to the Non-Diabetic) Population: A Systematic Review. PLoS One, 2016. 11(1): p. e0147329.

3. Isomaa, B., et al., Cardiovascular morbidity and mortality associated with the metabolic syndrome. Diabetes Care, 2001. 24(4): p. 683-9.

4. Giovannucci, E., et al., Diabetes and cancer: a consensus report. Diabetes Care, 2010. 33(7): p. 1674-85.

5. Younossi, Z.M., et al., Nonalcoholic fatty liver disease in patients with type 2 diabetes. Clin Gastroenterol Hepatol, 2004. 2(3): p. 262-5.

6. Adam, R., et al., Evolution of indications and results of liver transplantation in Europe. A report from the European Liver Transplant Registry (ELTR). J Hepatol, 2012. 57(3): p. 675-88.

7. Watt, K.D., Keys to long-term care of the liver transplant recipient. Nat Rev Gastroenterol Hepatol, 2015. 12(11): p. 639-48.

8. Neuberger, J., Liver transplantation in the United Kingdom. Liver Transpl, 2016. 22(8): p. 112935 .

9. Starzl, T.E., et al., Factors in Successful Renal Transplantation. Surgery, 1964. 56: p. 296-318.

10. Davidson, J.A., A. Wilkinson, and T. International Expert Panel on New-Onset Diabetes after, New-Onset Diabetes After Transplantation 2003 International Consensus Guidelines: an endocrinologist's view. Diabetes Care, 2004. 27(3): p. 805-12.

11. Pham, P.T., et al., New onset diabetes after transplantation (NODAT): an overview. Diabetes Metab Syndr Obes, 2011. 4: p. 175-86.

12. Marchetti, P., New-onset diabetes after liver transplantation: from pathogenesis to management. Liver Transpl, 2005. 11(6): p. 612-20.

13. Bloom, R.D. and M.F. Crutchlow, New-onset diabetes mellitus in the kidney recipient: diagnosis and management strategies. Clin J Am Soc Nephrol, 2008. 3 Suppl 2: p. S38-48.

14. Zelle, D.M., et al., Pancreatic beta-cell dysfunction and risk of new-onset diabetes after kidney transplantation. Diabetes Care, 2013. 36(7): p. 1926-32.

15. Song, J.L., et al., Minimizing tacrolimus decreases the risk of new-onset diabetes mellitus after liver transplantation. World J Gastroenterol, 2016. 22(6): p. 2133-41.

16. Moon, J.I., et al., Negative impact of new-onset diabetes mellitus on patient and graft survival after liver transplantation: Long-term follow up. Transplantation, 2006. 82(12): p. 1625-8.

17. Ling, Q., et al., New-onset diabetes after liver transplantation: a national report from China Liver Transplant Registry. Liver Int, 2016. 36(5): p. 705-12.

18. Lv, C., et al., New-onset diabetes after liver transplantation and its impact on complications and patient survival. J Diabetes, 2015. 7(6): p. 881-90.

19. Younossi, Z.M., et al., The impact of type 2 diabetes and obesity on the long-term outcomes of more than 85000 liver transplant recipients in the US. Aliment Pharmacol Ther, 2014. 40(6): $p$. 686-94.

20. Bhat, V., et al., New-Onset Diabetes and Preexisting Diabetes Are Associated With Comparable Reduction in Long-Term Survival After Liver Transplant: A Machine Learning Approach. Mayo Clin Proc, 2018. 93(12): p. 1794-1802. 
21. Liu, F.C., et al., Prevalence, predictive factors, and survival outcome of new-onset diabetes after liver transplantation: A population-based cohort study. Medicine (Baltimore), 2016. 95(25): p. e3829.

22. John, P.R. and P.J. Thuluvath, Outcome of patients with new-onset diabetes mellitus after liver transplantation compared with those without diabetes mellitus. Liver Transpl, 2002. 8(8): p. 708-13.

23. Yagi, S., et al., New-onset diabetes mellitus after living-donor liver transplantation: association with graft synthetic function. Surg Today, 2017. 47(6): p. 733-742.

24. Davidson, J., et al., New-onset diabetes after transplantation: 2003 International consensus guidelines. Proceedings of an international expert panel meeting. Barcelona, Spain, 19 February 2003. Transplantation, 2003. 75(10 Suppl): p. SS3-24.

25. Hartog, $\mathrm{H}_{\text {., }}$ et al., Early occurrence of new-onset diabetes after transplantation is related to type of liver graft and warm ischaemic injury. Liver Int, 2015. 35(6): p. 1739-47.

26. Saliba, F., et al., Risk factors for new-onset diabetes mellitus following liver transplantation and impact of hepatitis C infection : an observational multicenter study. Liver Transpl, 2007. 13(1): p. 136-44.

27. Vaughn, V.M., et al., Analytic morphomics identifies predictors of new-onset diabetes after liver transplantation. Clin Transplant, 2015. 29(5): p. 458-64.

28. Xue, M., et al., Donor liver steatosis: A risk factor for early new-onset diabetes after liver transplantation. J Diabetes Investig, 2017. 8(2): p. 181-187.

29. Abe, T., et al., Risk factors for development of new-onset diabetes mellitus and progressive impairment of glucose metabolism after living-donor liver transplantation. Transplant Proc, 2014. 46(3): p. 865-9.

30. Carey, E.J., et al., Pretransplant fasting glucose predicts new-onset diabetes after liver transplantation. J Transplant, 2012. 2012: p. 614781.

31. Li, Z., et al., New-Onset Diabetes Mellitus in Liver Transplant Recipients With Hepatitis C: Analysis of the National Database. Transplant Proc, 2016. 48(1): p. 138-44.

32. Cho, Y., et al., Statin therapy is associated with the development of new-onset diabetes after transplantation in liver recipients with high fasting plasma glucose levels. Liver Transpl, 2014. 20(5): p. 557-63.

33. Yadav, A.D., et al., New Onset Diabetes Mellitus in Living Donor versus Deceased Donor Liver Transplant Recipients: Analysis of the UNOS/OPTN Database. J Transplant, 2013. 2013: p. 269096.

34. Li, D.W., et al., Risk factors for new onset diabetes mellitus after liver transplantation: A metaanalysis. World J Gastroenterol, 2015. 21(20): p. 6329-40.

35. Syed, N.A. and R.L. Khandelwal, Reciprocal regulation of glycogen phosphorylase and glycogen synthase by insulin involving phosphatidylinositol-3 kinase and protein phosphatase-1 in HepG2 cells. Mol Cell Biochem, 2000. 211(1-2): p. 123-36.

36. Bussiere, C.T., et al., The impact of the mTOR inhibitor sirolimus on the proliferation and function of pancreatic islets and ductal cells. Diabetologia, 2006. 49(10): p. 2341-9.

37. Weir, M.R. and J.C. Fink, Risk for posttransplant Diabetes mellitus with current immunosuppressive medications. Am J Kidney Dis, 1999. 34(1): p. 1-13.

38. Forbes, J.M. and M.E. Cooper, Mechanisms of diabetic complications. Physiol Rev, 2013. 93(1): p. 137-88. 
39. Nusca, A., et al., Glycemic Variability in the Development of Cardiovascular Complications in Diabetes. Diabetes Metab Res Rev, 2018: p. e3047.

40. Park, S.C., et al., Effect of transient post-transplantation hyperglycemia on the development of diabetes mellitus and transplantation outcomes in kidney transplant recipients. Transplant Proc, 2015. 47(3): p. 666-71.

41. Cotovio, P., et al., New-onset diabetes after transplantation: assessment of risk factors and clinical outcomes. Transplant Proc, 2013. 45(3): p. 1079-83.

42. Alagbe, S.C., et al., New-onset diabetes after transplant: Incidence, risk factors and outcome. S Afr Med J, 2017. 107(9): p. 791-796.

43. Israni, A.K., et al., Clinical diagnosis of metabolic syndrome: predicting new-onset diabetes, coronary heart disease, and allograft failure late after kidney transplant. Transpl Int, 2012. 25(7): p. 748-57.

44. Klomjit, N., et al., Impact of Diabetes Mellitus on Survival Outcome of Lung Transplant Recipients: An Analysis of OPTN/UNOS Data. Clin Transpl, 2015. 31: p. 43-55. 
TABLES

Table 1: Demographic characteristics at liver transplantation of all patients together and of individual groups based on their state of diabetes mellitus.

\begin{tabular}{|c|c|c|c|c|c|}
\hline & $\begin{array}{l}\text { All patients }(\mathrm{n}=\mathbf{2}, 209) \\
\text { median }(\mathrm{IQR}) / \text { number }(\%)\end{array}$ & $\begin{array}{l}\text { Pre-DM group }(\mathrm{n}=\mathbf{2 9 8}) \\
\text { median }(\mathrm{IQR}) / \text { number }(\%)\end{array}$ & $\begin{array}{l}\text { No DM group }(n=1,431) \\
\text { median (IQR)/number }(\%)\end{array}$ & $\begin{array}{l}\text { PTDM group }(\mathbf{n = 3 6 2 )} \\
\text { median }(\text { IQR }) / \text { number }(\%)\end{array}$ & $\begin{array}{c}\text { t-HG group }(\mathrm{n}=\mathbf{1 1 8}) \\
\text { median }(\mathrm{IOR}) / \text { number }(\%)\end{array}$ \\
\hline Age (years) & $54(47-60)$ & $57(50-61)$ & $53(45-59)$ & $56(49-61)$ & $53(49-60)$ \\
\hline Male sex & $1480(67 \%)$ & $232(78 \%)$ & $899(63 \%)$ & $261(72 \%)$ & $88(75 \%)$ \\
\hline $\mathrm{BMI}\left(\mathrm{kg} / \mathrm{m}^{2}\right)$ & $26.4(23.3-30.3)$ & $27.4(24.4-31.4)$ & $25.9(22.8-29.8)$ & $27.4(24.4-31.8)$ & $27.0(24.6-30.4)$ \\
\hline $\mathrm{BMI}>30$ & $503(23 \%)$ & $89(30 \%)$ & $284(20 \%)$ & $98(27 \%)$ & $32(27 \%)$ \\
\hline$\Delta \mathrm{BMI}$ at $1^{\text {st }}$ year & $-0.29(-2.36-1.88)$ & $-0.04(-2.25-2.45)$ & $-0.26(-2.26-1.77)$ & $-0.32(-2.67-1.78)$ & $-1.45(-3.95-0.94)$ \\
\hline \multicolumn{6}{|l|}{ Etiology } \\
\hline NAFLD & $224(10 \%)$ & $54(18 \%)$ & $105(7 \%)$ & $59(16 \%)$ & $6(5 \%)$ \\
\hline $\mathrm{HCV}$ & $752(34 \%)$ & $118(40 \%)$ & $453(32 \%)$ & $127(35 \%)$ & $54(46 \%)$ \\
\hline ALD & $333(15 \%)$ & $51(17 \%)$ & $202(14 \%)$ & $69(19 \%)$ & $16(14 \%)$ \\
\hline HBV & $227(10 \%)$ & $24(8 \%)$ & $153(11 \%)$ & $31(9 \%)$ & $19(16 \%)$ \\
\hline AlH & $73(3 \%)$ & $11(4 \%)$ & $46(3 \%)$ & $13(4 \%)$ & $3(3 \%)$ \\
\hline PBC & $136(6 \%)$ & $8(3 \%)$ & $112(8 \%)$ & $13(4 \%)$ & $3(3 \%)$ \\
\hline PSC & $196(9 \%)$ & $13(4 \%)$ & $154(11 \%)$ & $21(6 \%)$ & $8(7 \%)$ \\
\hline Other & $268(12 \%)$ & $19(6 \%)$ & $206(14 \%)$ & $29(8 \%)$ & $9(8 \%)$ \\
\hline MELD score & $16(11-23)$ & $15(11-22)$ & $16(11-24)$ & $17(12-23)$ & $15(10-25)$ \\
\hline \multicolumn{6}{|c|}{ Immunosuppression exposuret } \\
\hline Tacrolimus & $1499(68 \%)$ & $215(72 \%)$ & $950(66 \%)$ & $263(73 \%)$ & $71(60 \%)$ \\
\hline Cyclosporin & $763(35 \%)$ & $97(33 \%)$ & $478(33 \%)$ & $150(41 \%)$ & $38(32 \%)$ \\
\hline Sirolimus & $157(7 \%)$ & $27(9 \%)$ & $78(5 \%)$ & $44(12 \%)$ & $8(7 \%)$ \\
\hline Prednisone & $450(20 \%)$ & $61(20 \%)$ & $285(20 \%)$ & $82(23 \%)$ & $22(19 \%)$ \\
\hline Mycophenolate & $1029(47 \%)$ & $149(50 \%)$ & $650(45 \%)$ & $184(51 \%)$ & $46(39 \%)$ \\
\hline
\end{tabular}

Please refer to the text for comparison between the individual groups. ta minimum of 6 months continuous exposure to an immunosuppressant was considered a positive exposure to that particular immunosuppressant.

Abbreviations: BMI body mass index; $\triangle \mathrm{BMI}$ change in $\mathrm{BMI} ; \mathrm{AlH}$ autoimmune hepatitis; ALD alcohol-related liver disease; HBV hepatitis $\mathrm{B} ; \mathrm{HCV}$ hepatitis $\mathrm{C}_{i}$ MELD model for end-stage liver disease; NAFLD non-alcoholic fatty liver disease; PBC primary biliary cholangitis; PSC primary sclerosing cholangitis. 
Table 2: Predictors of post-transplant diabetes mellitus (PTDM) in liver transplant recipients univariate and multivariate logistic regression

\begin{tabular}{l|c|c|c|c}
\cline { 2 - 4 } & Univariate analysis & \multicolumn{2}{c}{ Multivariate analysis } \\
\cline { 2 - 4 } & p value & Odds ratio & $95 \% \mathrm{Cl}$ & p value \\
\hline Age at listing* & $<0.0001$ & - & - & 0.051 \\
\hline Male sex* & 0.001 & 1.56 & $1.11-2.20$ & 0.01 \\
\hline BMI at listing* & $<0.0001$ & 1.03 & $1.00-1.06$ & 0.03 \\
\hline$\Delta$ BMI at 15t year post-LT & 0.99 & & & 0.002 \\
\hline Etiology-NAFLD* & $<0.0001$ & 2.03 & $1.31-3.16$ & \\
\hline Etiology-HCV & 0.21 & & & 0.01 \\
\hline Hypertension* & 0.07 & 2.40 & $1.19-4.82$ & 0.18 \\
\hline Dyslipidemia* & 0.02 & - & - & \\
\hline Donor age & 0.81 & & & 0.13 \\
\hline Cold ischemia time* & 0.01 & - & & \\
\hline Warm ischemia time & 0.23 & & & 0.007 \\
\hline Duct anastomosis - Roux-en-Y* & 0.00002 & 0.60 & $0.41-0.87$ & $<0.001$ \\
\hline Immunosuppression - Tacrolimus* & 0.06 & 2.76 & $1.78-4.30$ & 0.02 \\
\hline Immunosuppression - Cyclosporin* & 0.009 & 1.68 & $1.07-2.63$ & $<0.001$ \\
\hline Immunosuppression - Sirolimus* & $<0.0001$ & 2.72 & $1.67-4.42$ & $<0.02$ \\
\hline Immunosuppression - Prednisone* & 0.02 & 1.47 & $1.06-2.02$ & 0.02 \\
\hline Immunosuppression - Mycophenolate* & 0.07 & - & - & 0.77 \\
\hline
\end{tabular}

Only those who were not diabetic at the time of transplantation (i.e. not including those with pre-transplantation diabetes) were included in this analysis. Parameters with a $p$-value $<0.10$ on univariate analysis were included in the multivariate analysis and these parameters are indicated by an asterisk $\left(^{*}\right)$. The Bonferroni-corrected level of significance in this analysis was $\mathrm{p}<0.0038$. Abbreviations: $\mathrm{BMI}$ body mass index; $\triangle \mathrm{BMI}$ change in $\mathrm{BMI} \mathrm{HCV}$ hepatitis $\mathrm{C}$; LT liver transplantation; NAFLD non-alcoholic fatty liver disease. 


\section{FIGURE LEGENDS}

Figure 1 - Glycosylated Hemoglobin levels

Comparison of annual glycosylated hemoglobin ( $\mathrm{HbA} 1 \mathrm{c}$ ) levels in patients with pre-existing diabetes mellitus and patients who developed post-transplant diabetes mellitus (PTDM).

\section{Figure 2 - Patient Survival}

Kaplan-Meier estimates of patient survival time following liver transplantation in patients who never developed diabetes mellitus (no DM; green line); patients with pre-transplant diabetes mellitus (PreDM; blue line); and patients who developed post-transplant diabetes mellitus (PTDM; red line). 


\section{SUPPLEMENTARY TABLES}

Supplementary Table 1: Distribution of major causes of death in patients with pre-transplant diabetes, post-transplant diabetes, and no diabetes

\begin{tabular}{|c|c|c|c|}
\hline Cause of death & $\begin{array}{c}\text { Pre-DM group }(n=298) \\
\text { number }(\%)\end{array}$ & $\begin{array}{c}\text { No DM group }(\mathrm{n}=\mathbf{1}, \mathbf{4 3 1}) \\
\text { number }(\%)\end{array}$ & $\begin{array}{c}\text { PTDM group }(\mathrm{n}=362) \\
\text { number }(\%)\end{array}$ \\
\hline Cancert & $23(7.7 \%)$ & $120(8.4 \%)$ & $39(10.8 \%)$ \\
\hline Graft failure $\ddagger$ & $17(5.7 \%)$ & $84(5.9 \%)$ & $20(5.5 \%)$ \\
\hline Sepsis/Infection* & $17(5.7 \%)$ & $56(3.9 \%)$ & $21(5.8 \%)$ \\
\hline MSOF & $13(4.4 \%)$ & $80(5.6 \%)$ & $16(4.4 \%)$ \\
\hline Cerebrovascular event & $7(2.3 \%)$ & $20(1.4 \%)$ & $7(1.9 \%)$ \\
\hline Cardiac - heart failure & $2(0.7 \%)$ & $11(0.8 \%)$ & $3(0.8 \%)$ \\
\hline Cardiac-MI & $5(1.7 \%)$ & $14(1.0 \%)$ & $3(0.8 \%)$ \\
\hline ESRF & $4(1.3 \%)$ & $7(0.5 \%)$ & $3(0.8 \%)$ \\
\hline Other & $1(0.3 \%)$ & $29(1.2 \%)$ & $1(0.3 \%)$ \\
\hline Unknowns & $12(4.0 \%)$ & $85(5.9 \%)$ & $21(5.8 \%)$ \\
\hline
\end{tabular}

Of the total study population $(n=2,209), 782$ died following transplantation. This includes 101 deaths in the pre-transplant diabetes (pre-DM) group, 134 deaths in the post-transplant diabetes (PTDM) group, 506 deaths in the no diabetes gno DM) group, and 41 in the transient hyperglycemia group. tcancer deaths included those who died of recurrence of hepatocellular carcinoma. ¥graft failure deaths were due to causes such as recurrence of primary liver disease (e.g. hepatic $C$, autoimmune liver diseases, alcoholic recidivism), vascular complications, and graft rejection. *sepsis/infection deaths included acute infections (e.g. pneumonia) and chronic infections (e.g. invasive fungal infections. Sunknown cause of death also included those where the cause of death was documented as 'cardiac arrest' or 'respiratory arrest' or 'cardiorespiratory arrest/failure'. Abbreviations: DM diabetes mellitus; PTDM post-transplant diabetes mellitus; MSOF multi system organ failure; MI myocardial infarction; ESRF end stage renal failure.

Supplementary Table 2: Distribution of different types of de novo cancers in patients with pretransplant diabetes, post-transplant diabetes, and no diabetes

\begin{tabular}{lccc}
\hline Type of cancer & $\begin{array}{c}\text { Pre-DM group }(\mathrm{n}=298) \\
\text { number }(\%)\end{array}$ & $\begin{array}{c}\text { No DM group }(\mathrm{n}=\mathbf{1 , 4 3 1 )} \\
\text { number }(\%)\end{array}$ & $\begin{array}{c}\text { PTDM group }(\mathrm{n}=\mathbf{3 6 2}) \\
\text { number }(\%)\end{array}$ \\
\hline Upper Gl & $1(0.3 \%)$ & $3(0.2 \%)$ & $0(0.0 \%)$ \\
\hline Pancreato-biliary & $2(0.7 \%)$ & $7(0.5 \%)$ & $1(0.3 \%)$ \\
\hline Colon & $4(1.3 \%)$ & $12(0.8 \%)$ & $3(0.8 \%)$ \\
\hline Lung & $2(0.7 \%)$ & $8(0.6 \%)$ & $3(0.8 \%)$ \\
\hline PTLD & $3(1.0 \%)$ & $22(1.5 \%)$ & $3(0.8 \%)$ \\
\hline Hematological (excl. PTLD) & $1(0.3 \%)$ & $5(0.3 \%)$ & $1(0.3 \%)$ \\
\hline Renal (KUB) tract & $3(1.0 \%)$ & $13(0.9 \%)$ & $4(1.1 \%)$ \\
\hline Prostate & $3(1.0 \%)$ & $24(1.7 \%)$ & $4(1.1 \%)$ \\
\hline Skin & $1(0.3 \%)$ & $2(0.1 \%)$ & $3(0.8 \%)$ \\
\hline Breast & $2(0.7 \%)$ & $2(0.1 \%)$ & $2(0.6 \%)$ \\
\hline Central nervous system & $0(0.0 \%)$ & $2(0.1 \%)$ & $0(0.0 \%)$ \\
\hline Cancer of unknown primary & $2(0.7 \%)$ & $9(0.6 \%)$ & $3(0.8 \%)$ \\
\hline Other & $2(0.7 \%)$ & $7(0.5 \%)$ & $6(1.7 \%)$ \\
\hline
\end{tabular}

Abbreviations: DM diabetes mellitus; PTDM post-transplant diabetes mellitus; Gl gastrointestinal; PTLD post-transplant lymphoproliferative disease; KUB kidney, ureter, and bladder. 